\title{
VARIATIONAL FORMULAE FOR THE CAPACITY INDUCED BY SECOND-ORDER ELLIPTIC DIFFERENTIAL OPERATORS
}

\author{
C. LANDIM
}

\begin{abstract}
We review recent progress in potential theory of second-order elliptic operators and on the metastable behavior of Markov processes.
\end{abstract}

There has been many recent progress in the potential theory of non-reversible Markov processes. We review in this article some of these advances. In Section 1, we present a brief historical overview of potential theory and we introduce the main notions which will appear throughout the article. In Section 2] we present two variational formulae for the capacity between two sets induced by second-order elliptic operators non necessarily selfadjoint. In the following three sections we present applications of these results. In Section 3. we discuss recurrence of Markov processes; in Section 4, we present a sharp estimate for the transition time between two wells in a dynamical system randomly perturbed; and in Section 5, we prove the metastable behavior of this process.

\section{Potential theory}

We present in this section a brief historical introduction to the Dirichlet principle. The interested reader will find in Kellogg's book [22] a full account and references.

From Newton's law of universal gravitation to Laplace's equation. In 1687, Newton enunciated the Law of universal gravitation which states that "every particle of matter in the universe attracts every other particle with a force whose direction is that of the line joining the two, and whose magnitude is directly as the product of their masses, and inversely as the square of their distance from each other". The magnitude $F$ of the force between two particules, one of mass $m_{1}$ situated at $x \in \mathbb{R}^{3}$ and one of mass $m_{2}$ situated at $y \in \mathbb{R}^{3}$ is thus given by

$$
F=\kappa \frac{m_{1} m_{2}}{\|x-y\|^{2}}
$$

where $\left\|\left(z_{1}, z_{2}, z_{3}\right)\right\|=\sqrt{z_{1}^{2}+z_{2}^{2}+z_{3}^{2}}$ stands for the Euclidean distance, and $\kappa$ for a constant which depends only on the units used. In order to avoid the constant $\kappa$ we choose henceforth the unit of force so that $\kappa=1$.

Once Newton's gravitation law has been formulated, it is natural to calculate the force exerted on a particle of unit mass by different types of bodies. Consider a body $\mathfrak{B}$ occupying a portion $\Omega$ of the space $\mathbb{R}^{3}$. Assume that its density $\rho$ at each point $z \in \Omega$ is well defined and that it is continuous and bounded as a function of $z$. By density at $z$ we mean the limit of the ratio between the mass of a portion containing $z$ and the volume of the portion, as the volume of the portion vanishes. By (1.1), the force at a point $x \in \mathbb{R}^{3}$ is given by

$$
F(x)=\int_{\Omega} \frac{z-x}{\|z-x\|^{3}} \rho(z) d z
$$


Note that the force is well defined in $\Omega$ because $x \mapsto\|x\|^{-2}$ is integrable in a neighborhood of the origin and we assumed the density $\rho$ to be bounded. Equation (1.2) defines, therefore, a vector field $F=\left(F_{1}, F_{2}, F_{3}\right): \mathbb{R}^{3} \rightarrow \mathbb{R}^{3}$.

The force field $F$ introduced in (1.2) turns out to be divergence free in $\Omega^{c}$ :

$$
(\nabla \cdot F)(x):=\sum_{j=1}^{3}\left(\partial_{x_{j}} F_{j}\right)(x)=0, \quad x \in \Omega^{c},
$$

where $\partial_{x_{j}}$ represents the partial derivative with respect to $x_{j}$. It is also conservative: Fix a point $x \in \mathbb{R}$, and let $\gamma:[0,1] \rightarrow \mathbb{R}^{3}$ be a smooth, closed path such that $\gamma(0)=\gamma(1)=x$. The integral of the force field along the cycle $\gamma$ is given by

$$
\int F \cdot d \gamma:=\sum_{j=1}^{3} \int F_{j}(\gamma) d \gamma_{j}=\sum_{j=1}^{3} \int_{0}^{1} F_{j}(\gamma(t)) \gamma_{j}^{\prime}(t) d t=0 .
$$

As the force field is conservative and the space is simply connected [any two paths with the same endpoints can be continuously deformed one into the other], we may associate a potential $V: \mathbb{R}^{3} \rightarrow \mathbb{R}$ to the vector field $F$. Fix a point $x_{0} \in \mathbb{R}^{3}$ and a constant $C_{0}$, and let

$$
V(x)=C_{0}+\int F \cdot d \gamma,
$$

where $\gamma$ is a continuous path from $x_{0}$ to $x$. The potential $V$ is well defined because the force field is conservative, and it is unique up to an additive constant. By requiring it to vanish at infinity, it becomes

$$
V(x)=-\int_{\mathbb{R}^{3}} \frac{1}{\|z-x\|} \rho(z) d z,
$$

and it is called the Newton potential of the measure $\rho(z) d z$. Moreover, if we represent by $\nabla V$ the gradient of $V, \nabla V=\left(\partial_{x_{1}} V, \partial_{x_{2}} V, \partial_{x_{3}} V\right)$,

$$
\nabla V=F \text {. }
$$

Hence, since the force field is divergence-free [equation (1.3)],

$$
\Delta V:=\nabla \cdot \nabla V=0 \quad \text { on } \Omega^{c},
$$

which is known as Laplace's differential equation.

This last identity provides an alternative way to compute the force field induced by a body whose density is unknown. Let $\mathfrak{B}$ be a body occupying a portion $\Omega$ of the space $\mathbb{R}^{3}$. Assume that $\Omega^{c}$ is a domain [open and connected] which has a smooth, simply connected boundary, denoted by $\partial \Omega$. Assume, further, that the force field $F$ exerted by the body $\mathfrak{B}$ can be measured at the boundary of $\Omega$. Fix a point $x_{0} \in \partial \Omega$, set $V\left(x_{0}\right)=0$, and extend the definition of $V$ to $\partial \Omega$ through equation (1.4). By [1.7), the potential $V$ solves the equation

$$
\begin{cases}(\Delta W)(x)=0, & x \in \Omega^{c}, \\ W(x)=V(x), & x \in \partial \Omega .\end{cases}
$$

To derive $F$, it remains to solve the linear equation (1.8) and to retrieve $F$ from $V$ by (1.6).

The problem of proving the existence of a function satisfying (1.8) or of finding it when it exists is known as the Dirichlet problem, or the first boundary problem of potential theory.

Dirichlet's principle. In 1850, Dirichlet proposed the following argument to prove the existence of a solution to (1.8). It is simpler to present it in the context of masses distributed along surfaces. If mass points disturb, on may think in terms of charges since, according to 
Coulomb's law, two point charges exert forces on each other which are given by Newton's law with the word mass replaced by charge, except that charges may attract or repel each other.

Consider a bounded domain $\Omega$ whose boundary, represented by $\partial \Omega$, is smooth. Let $\zeta$ be a surface density on $\partial \Omega$. By (1.5), the potential associated to this mass distribution is given by

$$
V(x)=-\int_{\partial \Omega} \frac{1}{\|z-x\|} \zeta(z) \sigma(d z),
$$

where $\sigma(d z)$ stands for the surface measure. The surface density can be recovered from the potential. By Theorem VI of Chapter VI in [22],

$$
\frac{\partial V}{\partial n_{+}}(x)-\frac{\partial V}{\partial n_{-}}(x)=-4 \pi \zeta(x)
$$

where $n_{+}$, resp. $n_{-}$, represents the outward, resp. inward, pointing unit normal vector to $\partial \Omega$.

Denote by $E(\zeta)$ the potential energy of the mass distribution $\zeta$. It corresponds to the total work needed to assemble the distribution from a state of infinite dispersion, and it is given by

$$
E(\zeta)=\frac{1}{2} \int_{\partial \Omega} V(x) \zeta(x) \sigma(d x)
$$

Since, by (1.9), the surface density can be expressed in terms of the potential, we may consider the energy as a function of the potential. After this replacement, as the potential satisfies Laplace's equation (1.7) on $(\partial \Omega)^{c}$, applying the divergence theorem, we obtain that

$$
E(V)=\frac{1}{8 \pi} \int_{\mathbb{R}^{3}}\|\nabla V(x)\|^{2} d x .
$$

It is a principle of physics that equilibrium is characterized by the least potential energy consistent with the constraints of the problem. Thus, to prove the existence of a solution of the differential equation (1.8), Dirichlet proposed to consider the variational problem

$$
\inf _{f} \int_{\mathbb{R}^{3}}\|(\nabla f)(x)\|^{2} d x
$$

where the infimum is carried over all smooth functions $f: \mathbb{R}^{3} \rightarrow \mathbb{R}$ such that $f=V$ on $\partial \Omega$.

Mathematicians objected to the argument at an early date, pointing that the infimum might not be attained at an element of the class of functions considered. Weierstrass gave an example showing that the principle was false, and in 1899, Hilbert provided conditions on the surface, the boundary values and the class of functions $f$ admitted, for which the Dirichlet principel could be proved.

Condenser capacity. In electrostatics, the capacity of an isolated conductor is the the total charge the conductor can hold at a given potential.

Let $\Omega_{1} \subset \Omega_{2}$ be bounded domains with smooth boundaries represented by $\Sigma_{1}, \Sigma_{2}$, respectively. Assume that the closure of $\Omega_{1}$ is contained in $\Omega_{2}$. Consider the potential which is equal to 1 at $\Omega_{1}, 0$ at $\Omega_{2}^{c}$, and which satisfies Laplace's equation on $\mathcal{R}=\Omega_{2} \backslash \bar{\Omega}_{1}$. Since $V$ satisfies Laplace's equation on $\left(\Sigma_{1} \cup \Sigma_{2}\right)^{c}$, this potential can be obtained from a surface distribution concentrated on $\Sigma_{1} \cup \Sigma_{2}$. The total mass [charge] on $\Sigma_{1}$ is given by

$$
\int_{\Sigma_{1}} \zeta(x) \sigma(d x)=-\frac{1}{4 \pi} \int_{\Sigma_{1}} \frac{\partial V}{\partial n_{+}}(x) \sigma(d x)
$$


where the last identity follows from (1.9) and from the fact that the inward derivative vanishes because $V$ is constant in $\Omega_{1}$. The condenser capacity of $\Omega_{1}$ relative to $\Omega_{2}$ is given by

$$
\operatorname{cap}\left(\Omega_{1}, \Omega_{2}^{c}\right)=-\frac{1}{4 \pi} \int_{\Sigma_{1}} \frac{\partial V}{\partial n_{+}}(x) \sigma(d x),
$$

The measure $\nu=-(1 / 4 \pi)\left(\partial V / \partial n_{+}\right)(x) \sigma(d x)$ on $\Sigma_{1}$ is called the harmonic measure. The capacity of $\Omega_{1}$ is obtained by letting $\Omega_{2}$ increase to $\mathbb{R}^{d}$.

As the potential $V$ is equal to 1 on $\Omega_{1}$ and 0 at $\Sigma_{2}$, we may insert $V(x)$ in the previous integral, add the integral of the same expression over $\Sigma_{2}$, and then use the divergence theorem and the fact that $V$ is harmonic on the annulus $\mathcal{R}=\Omega_{2} \backslash \Omega_{1}$ to conclude that the previous expression is equal to

$$
\frac{1}{4 \pi} \int_{\mathcal{R}}\|(\nabla V)(x)\|^{2} d x=\frac{1}{4 \pi} \inf _{f} \int_{\mathbb{R}^{3}}\|(\nabla f)(x)\|^{2} d x,
$$

where the infimum is carried over all smooth functions $f$ such that $f=1$ on $\Omega_{1}$ and $f=0$ on $\Omega_{2}^{c}$. This latter formula provides a variational formula for the capacity defined by (1.10), called the Dirichlet principle.

In the next section, we present two variational formulae for the capacity induced by a second-order elliptic operator which is not self-adjoint with respect to the stationary state [as it is the case of the Laplace operator with respect to the Lebesgue measure]. We then present some applications of the formulae.

\section{DIRICHLET AND THOMSON PRINCIPLES}

In this section, we extend the notion of capacity to the context of general second order differential operators not necessarily self-adjoint. We then provide two variational formulae for the capacity, the so-called Dirichlet and Thomson principles. We will not be precise on the smoothness conditions of the functions and of the boundary of the sets. The interested reader will find in the references rigorous statements.

To avoid integrability conditions at infinity, we state the Dirichlet and the Thomson principles on a finite cube with periodic boundary conditions. Fix $d \geq 1$, and denote by $\mathbb{T}^{d}=[0,1)^{d}$ the $d$-dimensional torus of length 1 . Denote by $a(x)$ a uniformly positivedefinite matrix whose entries $a_{i, j}$ are smooth functions: There exist $c_{0}>0$ such that for all $x \in \mathbb{T}^{d}, \xi \in \mathbb{R}^{d}$,

$$
\xi \cdot a(x) \xi \geq c_{0}\|\xi\|^{2},
$$

where $\eta \cdot \xi$ represents the scalar product in $\mathbb{R}^{d}$.

Generator. Denote by $\mathcal{L}$ the generator given by

$$
\mathcal{L} f=\nabla \cdot(a \nabla f)+b \cdot \nabla f,
$$

where $b: \mathbb{T}^{d} \rightarrow \mathbb{R}^{d}$ is a smooth vector field. By modifying the drift $b$ we could assume the matrix $a$ to be symmetric. We will not assume this condition for reasons which will become clear in the next sections. There exists a unique Borel probability measure such that $\mu \mathcal{L}=0$. This measure is absolutely continuous, $\mu(d x)=m(x) d x$, where $m$ is the unique solution to

$$
\nabla \cdot\left(a^{\dagger} \nabla m\right)-\nabla \cdot(b m)=0,
$$

where $a^{\dagger}$ stands for the transpose of $a$. For existence, uniqueness and regularity conditions of solutions of elliptic equations, we refer to [17]. Let $V(x)=-\log m(x)$, so that $m(x)=$ $e^{-V(x)}$. 
Throughout this section $A, B$ represent two closed, disjoint subsets of $\mathbb{T}^{d}$ which are the closure of open sets with smooth boundaries.

For such a set $A$, denote by $\mu_{A}(d x)$ the measure $m(x) \sigma(d x)$ on $\partial A$, where $\sigma(d x)$ represents the surface measure. Hence, for every smooth vector field $\varphi$,

$$
\oint_{\partial A} \varphi(x) \cdot n_{A}(x) \mu_{A}(d x)=\oint_{\partial A} \varphi(x) \cdot n_{A}(x) e^{-V(x)} \sigma(d x),
$$

where $n_{A}$ represents the inward normal vector to $\partial A$.

We may rewrite the generator $\mathcal{L}$ introduced in (2.2) as

$$
\mathcal{L} f=e^{V} \nabla \cdot\left(e^{-V} a \nabla f\right)+c \cdot \nabla f,
$$

where $c=b+a^{\dagger} \nabla V$. It follows from (2.3) that

$$
\nabla \cdot\left(e^{-V} c\right)=0 .
$$

This implies that the operator $c \cdot \nabla$ is skew-adjoint in $L_{2}(\mu)$ : for any smooth functions $f$, $g: \mathbb{T}^{d} \rightarrow \mathbb{R}$

$$
\int f c \cdot \nabla g d \mu=-\int g c \cdot \nabla f d \mu,
$$

and that for any open set $D$ with a smooth boundary,

$$
\oint_{\partial D} c \cdot n_{D} d \mu_{D}=\int_{\mathbb{T}^{d} \backslash D} e^{V} \nabla \cdot\left(e^{-V} c\right) d \mu=0 .
$$

In view of (2.6), the adjoint of $\mathcal{L}$ in $L_{2}(\mu)$, represented by $\mathcal{L}^{*}$, is given by

$$
\mathcal{L}^{*} f=e^{V} \nabla \cdot\left(e^{-V} a^{\dagger} \nabla f\right)-c \cdot \nabla f,
$$

while the symmetric part, denoted by $\mathcal{L}^{s}, \mathcal{L}^{s}=(1 / 2)\left(\mathcal{L}+\mathcal{L}^{*}\right)$, takes the form

$$
\mathcal{L}^{s} f=e^{V} \nabla \cdot\left(e^{-V} a_{s} \nabla f\right) .
$$

where $a_{s}$ stands for the symmetrization of the matrix $a: a_{s}=(1 / 2)\left[a+a^{\dagger}\right]$.

Capacity. Recall that $A, B$ are closed sets satisfying (2.4). Let

$$
\Omega=\mathbb{T}^{d} \backslash(A \cup B) .
$$

Denote by $h=h_{A, B}$, resp. $h^{*}=h_{A, B}^{*}$, the unique solutions to the linear elliptic equations

$$
\left\{\begin{array} { l l } 
{ \mathcal { L } h = 0 } & { \text { on } \Omega , } \\
{ h = \chi _ { A } } & { \text { on } A \cup B , }
\end{array} \quad \left\{\begin{array}{ll}
\mathcal{L}^{*} h=0 & \text { on } \Omega, \\
h^{*}=\chi_{A} & \text { on } A \cup B,
\end{array}\right.\right.
$$

where $\chi_{C}, C \subset \mathbb{T}^{d}$, represents the indicator function of the set $C$. The functions $h, h^{*}$ are called the equilibrium potentials between $A$ and $B$. A function $f$ such that $(\mathcal{L} f)(x)=0$ is said to harmonic at $x$. If it is harmonic at all points in some domain $\Omega$, it is said to be harmonic in $\Omega$.

By analogy to the electrostatic definition (1.10) of the capacity of a set, define the $c a$ pacity between the sets $A, B$ of $\mathbb{T}^{d}$ as

$$
\operatorname{cap}(A, B):=\oint_{\partial A} a \nabla h \cdot n_{A} d \mu_{A}, \quad \operatorname{cap}^{*}(A, B):=\oint_{\partial A} a^{\dagger} \nabla h^{*} \cdot n_{A} d \mu_{A} .
$$

Since $h=1$ at $\partial A$ and $h=0$ at $\partial B$, we may insert $h$ in the integral and add the surface integral of the same expression over $\partial B$. Applying then the divergence theorem, we obtain that

$$
\operatorname{cap}(A, B)=\int_{\Omega} \nabla h \cdot a \nabla h d \mu+\int_{\Omega} h e^{V} \nabla \cdot\left(e^{-V} a \nabla h\right) d \mu .
$$


As $\nabla h$ vanishes on $A \cup B$, we may extend the integrals to $\mathbb{T}^{d}$. The integrand in the second term can be written as $h[\mathcal{L} h-c \cdot \nabla h]$. Since $c \cdot \nabla$ is skew-adjoint and $h$ is harmonic on $(\partial A \cup \partial B)^{c}$, we conclude that

$$
\operatorname{cap}(A, B)=\int \nabla h \cdot a \nabla h d \mu, \quad \operatorname{cap}^{*}(A, B)=\int \nabla h_{A, B}^{*} \cdot a^{\dagger} \nabla h_{A, B}^{*} d \mu .
$$

In the previous formulae, we may replace $a, a^{\dagger}$ by their symmetric part $a_{s}$, and we may restrict the integrals to $\Omega$.

Lemma 2.1. Let $A, B$ be two closed subsets satisfying the conditions (2.4). Then,

$$
\operatorname{cap}(A, B)=\operatorname{cap}(B, A)=\operatorname{cap}^{*}(A, B) .
$$

Moreover,

$$
\operatorname{cap}(A, B)=\oint_{\partial A}(a \nabla h+h c) \cdot n_{A} d \mu_{A} .
$$

Proof. It is clear that $\operatorname{cap}(B, A)=\operatorname{cap}(A, B)$ since $h_{B, A}=1-h_{A, B}$ as $A \cap B=\varnothing$. The proof of (2.12) is similar to the one which led from the definition of the capacity to (2.11). One has just to recall from (2.5) that $\nabla \cdot\left(e^{-V} c\right)=0$.

We turn to the proof that $\operatorname{cap}(A, B)=\operatorname{cap}^{*}(A, B)$ It relies on the claim that

$$
\operatorname{cap}(A, B)=\int_{\mathbb{T}^{d}}\left\{\nabla h^{*} \cdot a \nabla h-h^{*} c \cdot \nabla h\right\} d \mu=\int_{\mathbb{T}^{d}}\left\{\nabla h \cdot a^{\dagger} \nabla h^{*}+h c \cdot \nabla h^{*}\right\} d \mu .
$$

To prove this claim, repeat the calculations carried out to derive (2.12) to conclude that

$$
\int_{\mathbb{T}^{d}} \nabla h^{*} \cdot a \nabla h d \mu=\int_{\Omega} \nabla h^{*} \cdot a \nabla h d \mu=\oint_{\partial A} a \nabla h \cdot n_{A} d \mu_{A}+\int_{\Omega} h^{*} c \cdot \nabla h d \mu .
$$

Since $\nabla h$ vanishes on $A \cup B$, we may carry the second integral over $\mathbb{T}^{d}$. This proves the first identity of the claim because the first term on the right hand side is equal to the capacity between $A$ and $B$.

The same computation inverting the roles of $h$ and $h^{*}$ gives that

$$
\int_{\mathbb{T}^{d}} \nabla h \cdot a^{\dagger} \nabla h^{*} d \mu=\operatorname{cap}^{*}(A, B)-\int_{\mathbb{T}^{d}} h c \cdot \nabla h^{*} d \mu .
$$

Compare this identity with the previous one. The left-hand sides coincide. As $c \cdot \nabla$ is skewadjoint, the second terms on the right-hand sides are also equal. Hence, $\operatorname{cap}(A, B)=$ $\operatorname{cap}^{*}(A, B)$ because the first term on the right-hand side of the penultimate equation is $\operatorname{cap}(A, B)$. The previous identity together with the fact that $\operatorname{cap}^{*}(A, B)=\operatorname{cap}(A, B)$ yields the second identity of the claim.

Considering $\mathcal{L}^{*}$ in place of $\mathcal{L}$ we obtain from the previous lemma that

$$
\operatorname{cap}^{*}(A, B)=\oint_{\partial A}\left(a^{\dagger} \nabla h^{*}-h^{*} c\right) \cdot n_{A} d \mu_{A}
$$

Variational formulae for the capacity. Let $\mathcal{F}$ be the Hilbert space of vector fields $\varphi$ : $\Omega \rightarrow \mathbb{R}^{d}$ endowed with the scalar product $\langle\cdot, \cdot\rangle$ given by:

$$
\langle\varphi, \psi\rangle:=\int_{\Omega} \varphi \cdot a_{s}^{-1} \psi d \mu .
$$

Let $\mathcal{F}_{\gamma}, \gamma \in \mathbb{R}$, be the closure in $\mathcal{F}$ of the space of smooth vector fields $\varphi \in \mathcal{F}$ such that

$$
\nabla \cdot\left(e^{-V} \varphi\right)=0, \quad \oint_{\partial A} \varphi \cdot n_{A} d \mu_{A}=-\gamma .
$$


Let $\mathcal{C}_{\alpha, \beta}, \alpha, \beta \in \mathbb{R}$, be the space of smooth functions $f: \Omega \rightarrow \mathbb{R}$ such that $f \equiv \alpha$ on $A$ and $f \equiv \beta$ on $B$. For $f \in \mathcal{C}_{\alpha, \beta}$ define

$$
\Psi_{f}:=a_{s} \nabla f, \quad \Phi_{f}:=a^{\dagger} \nabla f-f c .
$$

Note that

$$
\left\langle\Psi_{h}, \Psi_{h}\right\rangle=\int_{\Omega} \nabla h \cdot a_{s} \nabla h d \mu=\operatorname{cap}(A, B) .
$$

Lemma 2.2. For every $\varphi \in \mathcal{F}_{\gamma}$ and $f \in \mathcal{C}_{\alpha, 0}$ we have that

$$
\left\langle\Phi_{f}-\varphi, \Psi_{h}\right\rangle=\gamma+\alpha \operatorname{cap}(A, B) .
$$

Proof. Fix $\varphi \in \mathcal{F}_{\gamma}$ and $f \in \mathcal{C}_{\alpha, 0}$. By definition of $\Phi_{f}$,

$$
\left\langle\Phi_{f}-\varphi, \Psi_{h}\right\rangle=\int_{\Omega}\left(a^{\dagger} \nabla f-f c-\varphi\right) \cdot \nabla h d \mu .
$$

Writing $a^{\dagger} \nabla f \cdot \nabla h$ as $\nabla f \cdot a \nabla h$, and integrating by parts, since $f=\alpha$ on $\partial A$ and $f=0$ on $\partial B$, the previous term becomes

$$
-\int_{\Omega}\left(f e^{V} \nabla \cdot\left(e^{-V} a \nabla h\right)+f c+\varphi\right) \cdot \nabla h d \mu+\alpha \oint_{\partial A} a \nabla h \cdot n_{A} d \mu_{A} .
$$

By definition, the last integral is the capacity between $A$ and $B$, while the expression involving $f$ is equal to $-f \mathcal{L} h$. This expression vanishes because $h$ is $\mathcal{L}$-harmonic in $\Omega$. Hence, since $h=\chi_{A}$ on $\partial A \cup \partial B$, by an integration by part, the previous expression is equal

$$
\int_{\Omega} h e^{V} \nabla \cdot\left(e^{-V} \varphi\right) d \mu-\oint_{\partial A} \varphi \cdot n_{A} d \mu_{A}+\alpha \operatorname{cap}(A, B) .
$$

By (2.14), this expression is equal to $\gamma+\alpha \operatorname{cap}(A, B)$, as claimed.

Theorem 2.3 (Dirichlet principle). Fix two disjoint subsets $A, B$ of $\mathbb{T}^{d}$ satisfying (2.4). Then,

$$
\operatorname{cap}(A, B)=\inf _{f \in \mathcal{C}_{1,0}} \inf _{\varphi \in \mathcal{F}_{0}}\left\langle\Phi_{f}-\varphi, \Phi_{f}-\varphi\right\rangle,
$$

and the infimum is attained for $h_{\star}=(1 / 2)\left(h+h^{*}\right)$ and $\varphi_{\star}=\Phi_{h_{\star}}-\Psi_{h}$.

Proof. Fix $f \in \mathcal{C}_{1,0}$ and $\varphi \in \mathcal{F}_{0}$. By Lemma2.2, applied with $\gamma=0$ and $\alpha=1$, and by Schwarz inequality,

$$
\operatorname{cap}(A, B)^{2}=\left\langle\Phi_{f}-\varphi, \Psi_{h}\right\rangle^{2} \leq\left\langle\Phi_{f}-\varphi, \Phi_{f}-\varphi\right\rangle\left\langle\Psi_{h}, \Psi_{h}\right\rangle .
$$

By (2.15), the last term is equal to $\operatorname{cap}(A, B)$, so that $\operatorname{cap}(A, B) \leq\left\langle\Phi_{f}-\varphi, \Phi_{f}-\varphi\right\rangle$ for every $f$ in $\mathcal{C}_{1,0}$ and $\varphi$ in $\mathcal{F}_{0}$.

Recall from the statement of the theorem the definition of $h_{\star}$ and $\varphi_{\star}$. Since $\Phi_{h_{\star}}-\varphi_{\star}=$ $\Psi_{h}$, by (2.15), $\operatorname{cap}(A, B)=\left\langle\Phi_{h_{\star}}-\varphi_{\star}, \Phi_{h_{\star}}-\varphi_{\star}\right\rangle$. Therefore, to complete the proof of the theorem, it remains to check that $h_{\star}$ belongs to $\mathcal{C}_{1,0}$, and $\varphi_{\star}$ to $\mathcal{F}_{0}$. It is easy to check the first condition. For the second one, observe that

$$
\nabla \cdot\left(e^{-V} \varphi_{\star}\right)=\frac{1}{2} e^{-V}\left(\mathcal{L}^{*} h^{*}-\mathcal{L} h\right)-\frac{1}{2}\left(h+h^{*}\right) \nabla \cdot\left(e^{-V} c\right) .
$$

This expression vanishes on $\Omega$ by the harmonicity of $h, h^{*}$ and in view of $(2.5)$. On the other hand,

$\oint_{\partial A} \varphi_{\star} \cdot n_{A} d \mu_{A}=\frac{1}{2} \oint_{\partial A}\left(a^{\dagger} \nabla h^{*}-h^{*} c\right) \cdot n_{A} d \mu_{A}-\frac{1}{2} \oint_{\partial A}(a \nabla h+h c) \cdot n_{A} d \mu_{A}$.

By Lemma 2.1 and identity (2.13), the previous expression is equal to $(1 / 2)\left\{\operatorname{cap}^{*}(A, B)-\right.$ $\operatorname{cap}(A, B)\}=0$, which completes the proof of the theorem. 
Theorem 2.4 (Thomson principle). Fix two disjoint subsets $A, B$ of $\mathbb{T}^{d}$ satisfying (2.4). Then,

$$
\frac{1}{\operatorname{cap}(A, B)}=\inf _{\varphi \in \mathcal{F}_{1}} \inf _{f \in \mathcal{C}_{0,0}}\left\langle\Phi_{f}-\varphi, \Phi_{f}-\varphi\right\rangle .
$$

Moreover, the infimum is attained at $h_{\star}=\left(h-h^{*}\right) / 2 \operatorname{cap}(A, B)$ and $\varphi_{\star}=\Phi_{h_{\star}}-$ $\Psi_{h / \operatorname{cap}(A, B)}$.

Proof. Fix $\varphi$ in $\mathcal{F}_{1}$ and $f$ in $\mathcal{C}_{0,0}$. By Lemma 2.2 (applied with $\alpha=0$ and $\gamma=1$ ), by Schwarz inequality, and by (2.15),

$1=\left\langle\Phi_{f}-\varphi, \Psi_{h}\right\rangle^{2} \leq\left\langle\Phi_{f}-\varphi, \Phi_{f}-\varphi\right\rangle\left\langle\Psi_{h}, \Psi_{h}\right\rangle=\left\langle\Phi_{f}-\varphi, \Phi_{f}-\varphi\right\rangle \operatorname{cap}(A, B)$.

By definition of $h_{\star}, \varphi_{\star}, \Phi_{h_{\star}}-\varphi_{\star}=\Psi_{h / \operatorname{cap}(A, B)}$, so that by (2.15),

$$
\left\langle\Phi_{h_{\star}}-\varphi_{\star}, \Phi_{h_{\star}}-\varphi_{\star}\right\rangle=\left\langle\Psi_{h / \operatorname{cap}(A, B)}, \Psi_{h / \operatorname{cap}(A, B)}\right\rangle=1 / \operatorname{cap}(A, B) .
$$

It remains to check that $h_{\star} \in \mathcal{C}_{0,0}$, and $\varphi_{\star} \in \mathcal{F}_{1}$. It is easy to verify the first condition. For the second one, observe that

$$
\varphi_{\star}=\frac{-1}{2 \operatorname{cap}(A, B)}\left\{[a \nabla h+h c]+\left[a^{\dagger} \nabla h^{*}-h^{*} c\right]\right\} .
$$

Therefore, $2 \operatorname{cap}(A, B) \nabla \cdot\left(e^{-V} \varphi_{\star}\right)=-e^{-V}\left[\mathcal{L} h+\mathcal{L}^{*} h^{*}\right]=0$ on $\Omega$. Moreover,

$$
\begin{aligned}
- & 2 \operatorname{cap}(A, B) \oint_{\partial A} \varphi_{\star} \cdot n_{A} d \mu_{A} \\
& =\oint_{\partial A}(a \nabla h+h c) \cdot n_{A} d \mu_{A}+\oint_{\partial A}\left(a^{\dagger} \nabla h^{*}-h^{*} c\right) \cdot n_{A} d \mu_{A} .
\end{aligned}
$$

By Lemma 2.1 and (2.13), the right-hand side is equal to $\operatorname{cap}(A, B)+\operatorname{cap}^{*}(A, B)=$ $2 \operatorname{cap}(A, B)$. This proves that $\varphi_{\star}$ belongs to $\mathcal{F}_{1}$, and completes the proof of the theorem.

Reversible case. In the reversible case, $c=0, a$ symmetric, the optimal flow $\varphi$ in the Dirichlet principle is the null one, so that

$$
\operatorname{cap}(A, B)=\inf _{f \in \mathcal{C}_{1,0}}\left\langle\Phi_{f}, \Phi_{f}\right\rangle=\inf _{f \in \mathcal{C}_{1,0}} \int_{\Omega} \nabla f \cdot a \nabla f d \mu .
$$

In the last identity we used the fact that $\Phi_{f}=\Psi_{f}=a \nabla f$. We thus recover the Dirichlet principle in the reversible context.

Similarly, in the reversible case, the optimal function $f$ in the Thomson principle is the null one, so that

$$
\frac{1}{\operatorname{cap}(A, B)}=\inf _{\varphi \in \mathcal{F}_{1}}\langle\varphi, \varphi\rangle
$$

which is the Thomson's principle in the reversible case.

We conclude this subsection comparing the capacity induced by the generator $\mathcal{L}$ with the one induced by the symmetric part of the generator, $\mathcal{L}^{s}$ given by $(2.8)$.

Fix two disjoint subsets $A, B$ satisfying (2.4). Denote by $\operatorname{cap}_{s}(A, B)$, the capacity between $A$ and $B$ induced $\mathcal{L}^{s}$. Since $h$ belongs to $\mathcal{C}_{1,0}$, by (2.11) and (2.16),

$$
\operatorname{cap}_{s}(A, B) \leq \operatorname{cap}(A, B) \text {. }
$$

In the case of Markov chains taking value in a countable state-space, it is proved in Lemma 2.6 of [16] that if the generator satisfies a sector condition with constant $C_{0}$,

$$
\left(\int(\mathcal{L} f) g d \mu\right)^{2} \leq C_{0} \int(-\mathcal{L} f) f d \mu \int(-\mathcal{L} g) g d \mu
$$


for all smooth functions $f, g$, then $\operatorname{cap}(A, B) \leq C_{0} \operatorname{cap}_{s}(A, B)$.

Stochastic representation. The operators $\mathcal{L}$ and $\mathcal{L}^{*}$ are generators of Markov processes on $\mathbb{T}^{d}$ with invariant measure $\mu$. More precisely, $\mathcal{L}$ is the generator of the solution of the stochastic differential equation

$$
d X_{t}=\left\{-a^{\dagger} \nabla V+\nabla \cdot a+c\right\}\left(X_{t}\right) d t+\sqrt{2 a_{s}} d W_{t},
$$

where $W_{t}$ is a standard $d$-dimensional Brownian motion, $\sqrt{2 a_{s}}$ represents the symmetric, positive-definite square root of $2 a_{s}$, and $\nabla \cdot a$ is the vector field whose $j$-th coordinate is $(\nabla \cdot a)_{j}=\sum_{1 \leq i \leq d} \partial_{x_{i}} a_{i, j}$. For $\mathcal{L}^{*}$, one has to replace the drift in (2.17) by $-a \nabla V+$ $\nabla \cdot a^{\dagger}-c$.

Denote by $C\left([0,+\infty) ; \mathbb{T}^{d}\right)$ the space of continuous functions $X:[0,+\infty) \rightarrow \mathbb{T}^{d}$ endowed with the topology of locally uniform convergence. Let $\left\{\mathbb{P}_{x}: x \in \mathbb{T}^{d}\right\}$, resp. $\left\{\mathbb{P}_{x}^{*}: x \in \mathbb{T}^{d}\right\}$, be the probability measures on $C\left([0,+\infty) ; \mathbb{T}^{d}\right)$ induced by the Markov process associated to the generator $\mathcal{L}$, resp. $\mathcal{L}^{*}$, starting from $x$.

Denote by $H_{C}, C$ a closed subset of $\mathbb{T}^{d}$, the hitting time of $C$ :

$$
H_{C}=\inf \left\{t \geq 0: X_{t} \in C\right\} .
$$

Lemma 2.5. Let $C$ be the closure of an open set with smooth boundary. Consider two continuous functions $b, f: \mathbb{T}^{d} \rightarrow \mathbb{R}$. Let $u: \mathbb{T}^{d} \rightarrow \mathbb{R}$ be given by

$$
u(x):=\mathbb{E}_{x}\left[b\left(X\left(H_{C}\right)\right)+\int_{0}^{H_{C}} f(X(t)) d t\right] .
$$

Then, $u$ is the unique solution to

$$
\begin{cases}\mathcal{L} u=-f & \text { on } \mathbb{T}^{d} \backslash C, \\ u=b & \text { on } \partial C .\end{cases}
$$

This result provides a stochastic representation for the harmonic functions $h=h_{A, B}$, $h^{*}=h_{A, B}^{*}$ introduced previously:

$$
h(x)=\mathbb{P}_{x}\left[H_{A}<H_{B}\right], \quad h^{*}(x)=\mathbb{P}_{x}^{*}\left[H_{A}<H_{B}\right] .
$$

Harmonic measure. In view of the definition (2.10) of the capacity, define the probability measure $\nu \equiv \nu_{A, B}$ as the harmonic measure on $\partial A \cup \partial B$ conditioned to $\partial A$ as

$$
d \nu:=\frac{1}{\operatorname{cap}(A, B)} a^{\dagger} \nabla h^{*} \cdot n_{A} d \mu_{A} .
$$

Proposition 2.6. For each continuous function $f: \mathbb{T}^{d} \rightarrow \mathbb{R}$,

$$
\mathbb{E}_{\nu}\left[\int_{0}^{H_{B}} f\left(X_{t}\right) d t\right]=\frac{1}{\operatorname{cap}(A, B)} \int h^{*} f d \mu .
$$

Proof. Fix a continuous function $f$, and let $\Omega_{B}=\mathbb{T}^{d} \backslash B$. Denote by $u$ the unique solution of the elliptic equation (2.18) with $C=B, b \equiv 0$. In view of Lemma2.5 and by definition of the harmonic measure $\nu$, the left hand side of (2.19) is equal to

$$
\frac{1}{\operatorname{cap}(A, B)} \oint_{\partial A} u\left[a^{\dagger} \nabla h^{*}\right] \cdot n_{A} d \mu_{A} .
$$

The integral of the same expression at $\partial B$ vanishes because $u$ vanishes on $\partial B$. Hence, by the divergence theorem, this expression is equal to

$$
\frac{1}{\operatorname{cap}(A, B)} \int_{\Omega} e^{V} \nabla \cdot\left\{e^{-V}\left[a^{\dagger} \nabla h^{*}\right] u\right\} d \mu .
$$


Since the equilibrium potential $h^{*}$ is harmonic on $\Omega$, the previous equation is equal to

$$
\frac{1}{\operatorname{cap}(A, B)} \int_{\Omega} \nabla h^{*} a \nabla u d \mu+\frac{1}{\operatorname{cap}(A, B)} \int_{\Omega} u c \cdot \nabla h^{*} d \mu .
$$

Consider the first term. Since $\nabla h^{*}$ vanishes on $A$, we may extend the integral to $\Omega_{B}=$ $\mathbb{T}^{d} \backslash B$. By the divergence theorem and since the equilibrium potential $h^{*}$ vanishes on $\partial B$, this expression is equal to

$$
-\frac{1}{\operatorname{cap}(A, B)} \int_{\Omega_{B}} h^{*} e^{V} \nabla \cdot\left\{e^{-V} a \nabla u\right\} d \mu .
$$

As $\mathcal{L} u=-f$ on $\Omega_{B}$, this expression is equal to

$$
\frac{1}{\operatorname{cap}(A, B)} \int_{\Omega_{B}} h^{*} c \cdot \nabla u d \mu+\frac{1}{\operatorname{cap}(A, B)} \int_{\Omega_{B}} h^{*} f d \mu .
$$

Since the equilibrium potential $h^{*}$ vanishes on $B$, we may replace $\Omega_{B}$ by $\mathbb{T}^{d}$ in the last integral.

Up to this point we proved that the left-hand side of (2.19) is equal to

$$
\frac{1}{\operatorname{cap}(A, B)}\left\{\int_{\mathbb{T}^{d}} h^{*} f d \mu+\int_{\Omega_{B}} h^{*} c \cdot \nabla u d \mu+\int_{\Omega} u c \cdot \nabla h^{*} d \mu\right\} .
$$

Since $\nabla h^{*}$ vanishes on $A \cup B$ and $h^{*}$ on $B$, we may extend the last two integrals to $\mathbb{T}^{d}$. By (2.6), the sum of the last two terms vanishes, which completes the proof of the proposition.

Proposition 2.6 is due to Bovier, Eckhoff, Gayrard and Klein [9] for reversible Markov chains. A generalization to non-reversible chains can be found in [5]. A Dirichlet principle, as a double variational formula of type $\inf _{f} \sup _{g}$ involving functions, was proved by Pinsky [31, 32, 33] in the context of diffusions. It has been derived by Doyle [12] and by Gaudillière and L. for Markov chains [16]. The Dirichlet principle, stated in Theorem 2.3. appeared in [16] for Markov chains and is due to L., Mariani and Seo [27] in the context of diffusion processes. The Thomson principle, stated in Theorem 2.4, is due to Slowik [36] in the context of Markov chains and appeared in [27] for diffusions.

\section{RECURRENCE OF MARKOV CHAINS}

The capacity is an effective tool to prove the recurrence or transience of Markov processes whose stationary state are explicitly known.

Consider the following open problem. Let $\boldsymbol{X}=\left\{\left(X_{k}, Y_{k}\right): k \in \mathbb{Z}\right\}$, be a sequence of independent, identically distributed random variables such that $P\left[\left(X_{0}, Y_{0}\right)=( \pm 1, \pm 1)\right]=$ $1 / 4$ for all 4 combinations of signs. Given a random environment $\boldsymbol{X}$ consider the discretetime random walk on $\mathbb{Z}^{2}$ whose jump probabilities are given by

$$
p\left((j, k),\left(j+Y_{k}, k\right)\right)=p\left((j, k),\left(j, k+X_{j}\right)\right)=1 / 2 \text { for all }(j, k) \in \mathbb{Z}^{2} .
$$

Denote by $Z_{t}=\left(Z_{t}^{1}, Z_{t}^{2}\right)$ the position at time $t \in \mathbb{Z}$ of the random walk. Equation (3.1) states that in the horizontal line $\{(p, q): q=k\} Z$ only jumps from $(j, k)$ to $\left(j+Y_{k}, k\right)$ for all $j$. In other words, on each horizontal line the random walk is totally asymmetric, but the direction of the jumps may be differ from line to line. Similarly, on the vertical lines $\{(p, q): p=j\}$ the random walk is totally asymmetric and only jumps from $(j, k)$ to $\left(j, k+X_{j}\right)$. It is not known if this random walk is recurrent or not [almost surely with respect to the random environment]. 
Fix an environment $\boldsymbol{X}$, and let $\boldsymbol{P}_{(j, k)}^{\boldsymbol{X}}$ be the distribution of the random walk $Z$ which starts at time $t=0$ from $(j, k)$. Denote by $H_{0}^{+}$the return time to $0: H_{0}^{+}=\inf \{t \geq$ $\left.1: Z_{t}=0\right\}$. The random walk is recurrent if and only if $\boldsymbol{P}_{0}^{\boldsymbol{X}}\left[H_{0}^{+}=\infty\right]=0$. Let $\left\{B_{N}: N \geq 1\right\}$ be an increasing sequence of finite sets such that $\cup_{N} B_{N}=\mathbb{Z}^{2}$, and note that

$$
\boldsymbol{P}_{0}^{\boldsymbol{X}}\left[H_{0}^{+}=\infty\right]=\lim _{N \rightarrow \infty} \boldsymbol{P}_{0}^{\boldsymbol{X}}\left[H_{B_{N}^{c}}<H_{0}^{+}\right],
$$

where $H_{B_{N}^{c}}$ stands for the hitting time of $B_{N}^{c}$.

In the context of discrete-time Markov chains evolving on a countable state-space the capacity between two disjoint sets $A, B$ is given by

$$
\operatorname{cap}(A, B)=\sum_{x \in A} M(x) P_{x}\left[H_{B}<H_{A}^{+}\right],
$$

where $M$ represents the stationary state of the Markov chain and $H_{B}$, resp. $H_{A}^{+}$, the hitting time of the set $B$, resp. the return time to the set $A$.

By the previous identity, the right-hand side of (3.2) can be rewritten as

$$
\frac{1}{M_{\boldsymbol{X}}(0)} \lim _{N \rightarrow \infty} \operatorname{cap}_{\boldsymbol{X}}\left(\{0\}, B_{N}^{c}\right),
$$

where $M_{\boldsymbol{X}}$ represents the stationary state of the random walk. It is easy to show that $M_{\boldsymbol{X}}$ does not depend on the environment and is constant, $M_{\boldsymbol{X}}(z)=1$ for all $z \in \mathbb{Z}^{2}$.

In view of the Dirichlet principle, to prove that the random walk is recurrent, one needs to find a sequence of functions $f_{N}$ in $\mathcal{C}_{1,0}$ and of vector fields $\varphi_{N}$ in $\mathcal{F}_{0}$ [with $A=\{0\}$ $B=B_{N}^{c}$ and depending on the environment $\left.\boldsymbol{X}\right]$ for which $\left\langle\Phi_{f_{N}}-\varphi_{N}, \Phi_{f_{N}}-\varphi_{N}\right\rangle$ vanishes asymptotically.

This has not been achieved yet. However, this is the simplest way to prove that the symmetric, nearest-neighbor random walk on $\mathbb{Z}^{2}$ is recurrent $[p((j, k),(j, k \pm 1))=p((j, k),(j \pm$ $1, k))=1 / 4]$. In this case also $M(z)=1$ for all $z \in \mathbb{Z}^{2}$. Consider $B_{N}=\{-(N-$ $1), \ldots, N-1\}^{2}$, and set $\varphi_{N}=0, f_{N}(x)=1-\log |x|_{m} / \log N, x \in B_{N}$, where $|0|_{m}=1$, $|(j, k)|=\max \{|j|,|k|\}$. For these sequences,

$$
\left\langle\Phi_{f_{N}}-\varphi_{N}, \Phi_{f_{N}}-\varphi_{N}\right\rangle=\frac{1}{4} \sum_{j=1}^{2} \sum_{x \in \mathbb{Z}^{2}}\left[f_{N}\left(x+e_{j}\right)-f_{N}(x)\right]^{2} \leq \frac{C_{0}}{\log N},
$$

where $\left\{e_{1}, e_{2}\right\}$ stands for the canonical basis of $\mathbb{R}^{2}$ and $C_{0}$ for a finite constant independent of $N$. This proves that the 2-dimensional, nearest-neighbor, symmetric random walk is recurrent.

\section{EYRING-KRAMERS FORMULA FOR THE TRANSITION TIME}

We examine in this section the stochastic differential equation (2.17) as a small perturbation of a dynamical system $\dot{x}(t)=F(x(t))$, by introducing a small parameter $\epsilon>0$ in the equation.

To reduce the noise in (2.17), we substitute $\sqrt{2 a_{s}}$ in the second term of the right-hand side by $\sqrt{2 \epsilon a_{s}}$. At this point, to keep the structure of the equation, we have to replace in the first term $a$ by $\epsilon a$. To avoid the term $-a^{\dagger} \nabla V$ to become small, we change $V$ to $V / \epsilon$. After these modifications the equation (2.17) becomes

$$
d X_{t}^{\epsilon}=\left\{-a^{\dagger} \nabla V+\epsilon \nabla \cdot a+c\right\}\left(X_{t}^{\epsilon}\right) d t+\sqrt{2 \epsilon a_{s}} d W_{t} .
$$


The diffusion $X_{t}^{\epsilon}$ is a small perturbation of the dynamical system $\dot{x}(t)=-\left[a^{\dagger} \nabla V\right](x(t))+$ $c(x(t))$. For the equilibrium points of this ODE to be the critical points of $V$, we require $V$ to be a Lyapounov functional. This is the case if $c \cdot \nabla V=0$ on $\mathbb{T}^{d}$.

The generator of the diffusion $X_{t}^{\epsilon}$, denoted by $\mathcal{L}_{\epsilon}$, is given by

$$
\mathcal{L}_{\epsilon} f=\epsilon e^{V / \epsilon} \nabla \cdot\left\{e^{-V / \epsilon} a \nabla f\right\}+c \cdot \nabla f .
$$

Let $\mu_{\epsilon}$ be the probability measure given by

$$
\mu_{\epsilon}(d x)=\frac{1}{Z_{\epsilon}} \exp \{-V(x) / \epsilon\} d x
$$

where $Z_{\epsilon}$ is the normalizing constant, $Z_{\epsilon}:=\int_{\mathbb{T}^{d}} \exp \{-V(x) / \epsilon\} d x$. We have seen in the previous section that $\mu_{\epsilon}$ is the stationary state of the process $X_{t}^{\epsilon}$ provided $\nabla \cdot\left(e^{-V / \epsilon} c\right)=0$. Since $c \cdot \nabla V$ vanishes, this equation becomes $\nabla \cdot c=0$. We assume therefore that

$$
c \cdot \nabla V=0 \text { and } \nabla \cdot c=0 \text { on } \mathbb{T}^{d} .
$$

We examine the transition time in the case where $V$ is a double well potential. Assume that there exists an open set $\mathcal{G} \subset \mathbb{T}^{d}$ such that

(H1) The potential $V$ has a finite number of critical points in $\mathcal{G}$. Exactly two of them, denoted by $\boldsymbol{m}_{1}$ and $\boldsymbol{m}_{2}$, are local minima. The Hessian of $V$ at each of these minima has $d$ strictly positive eigenvalues.

(H2) There is one and only one saddle point between $\boldsymbol{m}_{1}$ and $\boldsymbol{m}_{2}$ in $\mathcal{G}$, denoted by $\boldsymbol{\sigma}$. The Hessian of $V$ at $\sigma$ has exactly one strictly negative eigenvalue and $(d-1)$ strictly positive eigenvalues.

(H3) We have that $V(\boldsymbol{\sigma})<\inf _{x \in \partial \mathcal{G}} V(x)$.

Assume without loss of generality that $V\left(\boldsymbol{m}_{2}\right) \leq V\left(\boldsymbol{m}_{1}\right)$, so that $\boldsymbol{m}_{2}$ is the global minimum of the potential $V$ in $\mathcal{G}$. Denote by $\Omega$ the level set of the potential defined by saddle point, $\Omega=\{x \in \mathcal{G}: V(x)<V(\boldsymbol{\sigma})\}$. Let $\mathcal{V}_{1}, \mathcal{V}_{2}$ be two domains with smooth boundary containing $\boldsymbol{m}_{1}$ and $\boldsymbol{m}_{2}$, respectively, and contained in $\Omega$ :

$$
\boldsymbol{m}_{i} \in \mathcal{V}_{i} \subset\{x \in \mathcal{G}: V(x)<V(\boldsymbol{\sigma})-\kappa\}
$$

for some $\kappa>0$.

Denote by $\nabla^{2} V(x)$ the Hessian of $V$ at $x$. By Lemma 10.1 of [28], both $\left[\nabla^{2} V a\right](\boldsymbol{\sigma})$ and $\left[\nabla^{2} V a^{\dagger}\right](\sigma)$ have a unique (and the same) negative eigenvalue. Denote by $-\mu$ this common negative eigenvalue.

Let $\mathbb{P}_{x}^{\epsilon}, x \in \mathbb{T}^{d}$, be the probability measure on $C\left(\mathbb{R}_{+}, \mathbb{T}^{d}\right)$ induced by the Markov process $X_{t}^{\epsilon}$ starting from $x$. Expectation with respect to $\mathbb{P}_{x}^{\epsilon}$ is represented by $\mathbb{E}_{x}^{\epsilon}$.

Theorem 4.1 (Eyring-Kramers formula). We have that

$$
\mathbb{E}_{\boldsymbol{m}_{1}}^{\epsilon}\left[H_{\mathcal{V}_{2}}\right]=\left[1+o_{\epsilon}(1)\right] \mathfrak{p} e^{\Lambda / \epsilon}, \quad \text { where } \mathfrak{p}=\frac{2 \pi}{\mu} \frac{\sqrt{-\operatorname{det}\left[\nabla^{2} V(\boldsymbol{\sigma})\right]}}{\sqrt{\operatorname{det}\left[\left(\nabla^{2} V\right)\left(\boldsymbol{m}_{1}\right)\right]}}
$$

and $\Lambda=V(\boldsymbol{\sigma})-V\left(\boldsymbol{m}_{1}\right)$.

The term $\mathfrak{p}$ is called the prefactor. It can be understood as the first-order term in the expansion in $\epsilon$ of the exponential barrier. Let $\mathbb{E}_{\boldsymbol{m}_{1}}^{\epsilon}\left[H_{\mathcal{V}_{2}}\right]=\exp \{\Lambda(\epsilon) / \epsilon\}$. Theorem 4.1 states that $\Lambda(\epsilon)=\Lambda+\epsilon \log \mathfrak{p}+o(\epsilon)$.

The proof of this theorem in the case $c=0$ and $a$ independent of $x, a(x)=a$, can be found in [27]. Uniqueness of local minima and of saddle points connecting the wells is not required there. The same argument should apply to the general case under the hypotheses (2.1), (4.3), but the proof has not been written. 
The 0-th order term in the expansion, $\Lambda$, can be obtained from Freidlin and Wentzell large deviations theory of random perturbations of dynamical systems [15]. The pre-factor $\mathfrak{p}$ has been calculated rigorously for reversible diffusions by Sugiura [37, 38] [based on asymptotics of the principal eigenvalue and eigenfunction for a Dirichlet boundary value problem in a bounded domain], and independently, by Bovier, Eckhoff, Gayrard and Klein [10] [based on potential theory]. We refer to [6] for a recent review.

In the context of chemical reactions, the transition time $\mathbb{E}_{\boldsymbol{m}_{1}}^{\epsilon}\left[H_{\mathcal{V}_{2}}\right]$ corresponds to the inverse of the rate of a reaction. The so-called "Arrenhuis law" relates the rate of a reaction to the absolute temperature. It seems to have been first discovered empirically by Hood [19]. van't Hoff [18] proposed a thermodynamical derivation of the law, and Arrhenius [1] physical arguments based on molecular dynamics. In the self-adjoint case, the pre-factor $\mathfrak{p}$ first appeared in Eyring [14] and in more explicit form in Kramers [23]. Bouchet and Reygnier [8] derived the formula in the non-reversible situation.

\section{Metastability}

We developed in these last years a robust method to prove the metastable behavior of Markov processes based on potential theory. We report in this section recent developments which rely on asymptotic properties of elliptic operators.

We first define metastability. Let $Z_{\epsilon}(t)$ be a sequence of Markov processes taking values in some space $E_{\epsilon}$. Let $\left\{\mathcal{E}_{\epsilon}^{1}, \ldots, \mathcal{E}_{\epsilon}^{n}, \Delta_{\epsilon}\right\}$ be a partition of the set $E_{\epsilon}$, and set $\mathcal{E}_{\epsilon}=\mathcal{E}_{\epsilon}^{1} \cup$ $\cdots \cup \mathcal{E}_{\epsilon}^{n}$.

Fix a sequence of positive numbers $\theta_{\epsilon}$, and denote by $\widehat{Z}_{\epsilon}(t)$ the process $Z_{\epsilon}(t)$ speededup by $\theta_{\epsilon}: \widehat{Z}_{\epsilon}(t)=Z_{\epsilon}\left(t \theta_{\epsilon}\right)$. Denote by $\widehat{\boldsymbol{P}}_{\epsilon, x}, x \in E_{\epsilon}$, the distribution of the process $\widehat{Z}_{\epsilon}(t)$ starting from $x$. Let $S=\{1, \ldots, n\}, S_{0}=\{0\} \cup S$, and let $\Upsilon_{\epsilon}: E_{\epsilon} \rightarrow S_{0}$ be the projection given by

$$
\Upsilon_{\epsilon}(x)=\sum_{j=1}^{n} j \chi_{\mathcal{E}_{\epsilon}^{j}}(x) .
$$

Note that points in $\Delta_{\epsilon}$ are mapped to 0 . Denote by $z_{\epsilon}(t)$ the $S_{0}$-valued process defined by

$$
z_{\epsilon}(t)=\Upsilon_{\epsilon}\left(\widehat{Z}_{\epsilon}(t)\right)=\Upsilon_{\epsilon}\left(Z_{\epsilon}\left(t \theta_{\epsilon}\right)\right) .
$$

The process $z_{\epsilon}(t)$ is usually not Markovian.

Definition 5.1. [Metastability]. We say that the process $Z_{\epsilon}(t)$ is metastable in the time scale $\theta_{\epsilon}$, with metastable sets $\mathcal{E}_{\epsilon}^{1}, \ldots, \mathcal{E}_{\epsilon}^{n}$ if there exists a $S$-valued, continuous-time Markov chain $z(t)$ such that for all $x \in \mathcal{E}_{\epsilon}$ the $\widehat{\boldsymbol{P}}_{\epsilon, x}$-finite-dimensional distributions of $z_{\epsilon}(t)$ converge to the finite-dimensional distributions of $z(t)$.

The Markov chain $z(t)$ is called the reduced chain. Mind that the reduced chain does not take the value 0 . The sojourns of $\widehat{Z}_{\epsilon}(t)$ at $\Delta_{\epsilon}$ are washed-out in the limit. Of course, the same process $Z_{\epsilon}(t)$ may exhibit different metastable behaviors in different time-scales or even different metastable behaviors in the same time-scale but in different regions of the space, inaccessible one to the other in that time-scale.

In some examples [20, 21, 2] the set $S$ may be countably infinite. In these cases $\Upsilon_{\epsilon}$ is a projection from $E_{\epsilon}$ to a finite set $S_{\epsilon} \cup\{0\}$, where $S_{\epsilon}$ increases to a countable set $S$, and we require $\# E_{\epsilon} / \# S_{\epsilon} \rightarrow 0$.

In the remaining part of this section we prove that under certain hypotheses the diffusion $X_{t}^{\epsilon}$ is metastable. Some of these conditions are not needed, but they simplify the presentation. The reader will find in the references finer results. 
We assume from now on that the potential $V$ fulfills the following set of assumptions. There exists an open set $\mathcal{G}$ of $\mathbb{T}^{d}$ such that

(H1') The function $V$ has a finite number of critical points in $\mathcal{G}$. The global minima of $V$ are represented by $m_{1}, \ldots, m_{n}$. They all belong to $\mathcal{G}$ and they are all at the same height: $V\left(m_{i}\right)=V\left(m_{j}\right)$ for all $i, j$. The Hessian of $V$ at each of these minima has $d$ strictly positive eigenvalues.

(H2') Denote by $\left\{\sigma_{1}, \ldots, \sigma_{\ell}\right\}$ the set of saddle points between the global minima. Assume that all saddle points are at the same height and that the Hessian of $V$ at these points has exactly one strictly negative eigenvalue and $(d-1)$ strictly positive eigenvalues.

(H3') We have that $V\left(\sigma_{1}\right)<\inf _{x \in \partial \mathcal{G}} V(x)$.

Denote by $\Omega$ the level set of the potential defined by the height of the saddle points: $\Omega=\left\{x \in \mathcal{G}: V(x)<V\left(\sigma_{1}\right)\right\}$. Let $\mathcal{W}_{1}, \ldots, \mathcal{W}_{p}$ be the connected components of $\Omega$. Assume that each of these sets contains one and only one global minima, so that $p=n$. Denote by $v_{1}, \ldots, \mathcal{V}_{n}$ domains with smooth boundaries satisfying (4.4) for $1 \leq i \leq n$, and let

$$
\mathcal{V}=\bigcup_{j=1}^{n} \mathcal{V}_{j}, \quad \Delta=\mathbb{T}^{d} \backslash \mathcal{V}, \quad \breve{\mathcal{V}}_{j}=\bigcup_{k: k \neq j} \mathcal{V}_{k}
$$

Recall from (4.5) the definition of $\Lambda$. Denote by $\widehat{X}_{t}^{\epsilon}$ the process $X_{t}^{\epsilon}$ speeded-up by $\theta_{\epsilon}=e^{\Lambda / \epsilon}$. This is the diffusion on $\mathbb{T}^{d}$ whose generator, denoted by $\widehat{\mathcal{L}}_{\epsilon}$, is given by $\widehat{\mathcal{L}_{\epsilon}}=\theta_{\epsilon} \mathcal{L}_{\epsilon}$. Denote by $\mathbb{P}_{x}^{\epsilon}$, resp. $\widehat{\mathbb{P}}_{x}^{\epsilon}, x \in \mathbb{T}^{d}$, the probability measure on $C\left(\mathbb{R}_{+}, \mathbb{T}^{d}\right)$ induced by the diffusion $X_{t}^{\epsilon}$, resp. $\widehat{X}_{t}^{\epsilon}$, starting from $x$. Expectation with respect to $\mathbb{P}_{x}^{\epsilon}$, is represented by $\mathbb{E}_{x}^{\epsilon}$.

Let $S=\{1, \ldots, n\}, S_{0}=\{0\} \cup S$. Denote by $\Upsilon: \mathbb{T}^{d} \rightarrow S_{0}$ the projection given by 5.1) with $\mathcal{E}_{\epsilon}^{j}$ replaced by $\mathcal{V}_{j}$, and let $x_{\epsilon}(t)$ be the $S_{0}$-valued process defined by

$$
x_{\epsilon}(t)=\Upsilon\left(\widehat{X}_{t}^{\epsilon}\right)=\Upsilon\left(X^{\epsilon}\left(t \theta_{\epsilon}\right)\right) .
$$

Note that $x_{\epsilon}(t)$ is not Markovian.

The proof of the metastable behavior of the diffusion $X_{t}^{\epsilon}$ is divided in four steps. We first show that in the time scale $\theta_{\epsilon}$ the process $X_{t}^{\epsilon}$ spends a negligible amount of time in the set $\Delta$. Then, we derive a candidate for the $S$-valued Markov chain which is supposed to describe the asymptotic behavior of the process among the wells. In the third step, we prove that the projection of the trace of $\widehat{X}_{t}^{\epsilon}$ on $\mathcal{V}$ converges to the $S$-valued Markov chain introduced in the second step. Finally, we show that the previous results together with an extra condition yield the convergence of the finite-dimensional distributions of $x_{\epsilon}(t)$.

Step 1: The set $\Delta$ is negligible. We first examine in the next lemma the time spent on the set $\Delta$.

Lemma 5.2. For all $t>0$,

$$
\lim _{\epsilon \rightarrow 0} \sup _{x \in \mathcal{V}} \mathbb{E}_{x}^{\epsilon}\left[\int_{0}^{t} \chi_{\Delta}\left(X\left(s \theta_{\epsilon}\right)\right) d s\right]=0 .
$$

Proof. Here is a sketch of the proof of this result which highlights the relevance of the variational formulae for the capacity. Denote by $\operatorname{cap}_{\epsilon}(\mathcal{A}, \mathcal{B})$ the capacity between two disjoint subsets $\mathcal{A}, \mathcal{B}$ with respect to the diffusion $X_{t}^{\epsilon}$.

Fix $1 \leq j \leq n$ and assume that $x$ belongs to $\nu_{j}$. The time scale $\theta_{\epsilon}$ is of the order of the transition time $H_{\breve{V}_{j}}$, where the $\breve{V}_{j}$ has been introduced in (5.2). The expectation appearing 
in the statement of the lemma is therefore of the same order of

$$
\frac{1}{\theta_{\epsilon}} \mathbb{E}_{x}^{\epsilon}\left[\int_{0}^{H_{\check{v}_{j}}} \chi_{\Delta}(X(s)) d s\right] \sim \frac{1}{\theta_{\epsilon} \operatorname{cap}_{\epsilon}\left(\mathcal{V}_{j}, \breve{V}_{j}\right)} \int_{\mathbb{T}^{d}} \chi_{\Delta} h_{\mathcal{v}_{j}, \breve{v}_{j}} d \mu_{\epsilon},
$$

where last step follows from Proposition 2.6. It would be an identity if we had the harmonic measure in place of the Dirac measure concentrated on $x$, but these expectations should not be very different because $x$ belongs to the basin of attraction of $m_{j}$. Since $\mu_{\epsilon}(\Delta) \rightarrow 0$, the proof is completed if we can show, using the variational principles, that $\theta_{\epsilon} \operatorname{cap}_{\epsilon}\left(\mathcal{V}_{j}, \breve{V}_{j}\right)$ converges to a positive value.

Step 2: The reduced chain. The time-scale $\theta_{\epsilon}$ at which the process $X_{t}^{\epsilon}$ evolves among the wells should be of the order of the transition time $\mathbb{E}_{m_{j}}^{\epsilon}\left[H\left(\breve{V}_{j}\right)\right]$. Hence, by Proposition 2.6 ,

$$
\theta_{\epsilon} \sim \mathbb{E}_{m_{j}}^{\epsilon}\left[H\left(\breve{\mathcal{V}}_{j}\right)\right] \sim \frac{1}{\operatorname{cap}_{\epsilon}\left(\mathcal{V}_{j}, \breve{\mathcal{V}}_{j}\right)} \int h_{\mathcal{V}_{j}, \breve{\mathcal{V}}_{j}^{*}} d \mu
$$

Since $m_{j}$ is a global minimum of $V$, the last integral is of order 1 because the harmonic function $h_{\mathcal{V}_{j}, \breve{v}_{j}^{*}}$ is equal to 1 at $\mathcal{V}_{j}$. We conclude that the time-scale $\theta_{\epsilon}$ should be of the order $\operatorname{cap}_{\epsilon}\left(\mathcal{V}_{j}, \breve{v}_{j}\right)^{-1}$.

It is proved in [3, 5], in the context of Markov chains taking values in a countable state space, that under certain assumptions

$$
\lambda_{j}:=\lim _{\epsilon \rightarrow 0} \theta_{\epsilon} \frac{1}{\mu_{\epsilon}\left(\mathcal{V}_{j}\right)} \operatorname{cap}_{\epsilon}\left(\mathcal{V}_{j}, \breve{\mathcal{V}}_{j}\right)
$$

represents the holding time at $j$ of the reduced chain. Moreover, in the reversible case, the jump rates $r(j, k)$ of the reduced chain are given by

$r(j, k)=\lim _{\epsilon \rightarrow 0} \frac{1}{2 \mu_{\epsilon}\left(\mathcal{V}_{j}\right)}\left\{\operatorname{cap}_{\epsilon}\left(\mathcal{V}_{j}, \breve{\mathcal{V}}_{j}\right)+\operatorname{cap}_{\epsilon}\left(\mathcal{V}_{k}, \breve{\mathcal{V}}_{k}\right)-\operatorname{cap}_{\epsilon}\left(\mathcal{V}_{j} \cup \mathcal{V}_{k}, \mathcal{V} \backslash\left[\mathcal{V}_{j} \cup \mathcal{V}_{k}\right]\right)\right\}$.

In the non-reversible case, the jump rates are more difficult to derive. By [5, Proposition 4.2], still in the context of Markov chains taking values in a countable state space,

$$
r(j, k)=\lambda_{j} \lim _{\epsilon \rightarrow 0} \overline{\mathbb{P}}_{m_{j}}^{\epsilon}\left[H\left(\mathcal{V}_{k}\right)<H\left(\mathcal{V} \backslash\left[\mathcal{V}_{j} \cup \mathcal{V}_{k}\right]\right)\right],
$$

where $\overline{\mathbb{P}}_{m_{j}}^{\epsilon}$ represents the distribution of the process in which the well $\mathcal{V}_{j}$ has been collapsed to the point $m_{j}$. Estimates on the harmonic function appearing on the right-hand of this equation are obtained by showing that this function solves a variational problem, similar to the one for the capacity, and then that to be optimal, a function has to take a precise value at the set $\mathcal{V}_{j}$. We refer to [24, 28] for details, where this program has been successfully undertaken for two different models.

Assume that one can compute the asymptotic jump rates through the previous formulae or that one can guess by other means the jump rates of the reduced chain. Denote by $\boldsymbol{L}$ the generator of the $S$-valued continuous-time Markov chain induced by these jump rates. Let $D\left(\mathbb{R}_{+}, E\right), E$ a metric space, be the space of $E$-valued, right-continuous functions with left-limits endowed with the Skorohod topology, and let $\boldsymbol{Q}_{j}, j \in S$, the measure on $D\left(\mathbb{R}_{+}, S\right)$ induced by the Markov chain with generator $\boldsymbol{L}$ starting from $j$.

Step 3: Convergence of the trace. We turn to the convergence of the trace process. Recall that $\widehat{X}_{t}^{\epsilon}$ represents the process $X_{t}^{\epsilon}$ speeded-up by $\theta_{\epsilon}$. Denote by $T_{\mathcal{V}}(t), t \geq 0$, the total 
time spent by the diffusion $\widehat{X}^{\epsilon}$ on the set $\mathcal{V}$ in the time interval $[0, t]$ :

$$
T_{\mathcal{V}}(t):=\int_{0}^{t} \chi_{\mathcal{V}}\left(\widehat{X}_{s}^{\epsilon}\right) d s
$$

Denote by $\left\{S_{\mathcal{V}}(t): t \geq 0\right\}$ the generalized inverse of $T_{\mathcal{V}}(t)$ :

$$
S_{\mathcal{V}}(t):=\sup \left\{s \geq 0: T_{\mathcal{V}}(s) \leq t\right\} .
$$

Clearly, for all $r \geq 0, t \geq 0$,

$$
\left\{S_{\mathcal{V}}(r) \geq t\right\}=\left\{T_{\mathcal{V}}(t) \leq r\right\}
$$

It is also clear that for any starting point $x \in \mathbb{T}^{d}, \lim _{t \rightarrow \infty} T_{\mathcal{V}}(t)=\infty$ almost surely. Therefore, the random path $\left\{Y_{\epsilon}(t): t \geq 0\right\}$, given by $Y_{\epsilon}(t):=\widehat{X}^{\epsilon}\left(S_{\mathcal{V}}(t)\right)$, is well defined for all $t \geq 0$ and takes value in the set $\mathcal{V}$. We call the process $Y_{\epsilon}(t)$ the trace of $\widehat{X}_{t}^{\epsilon}$ on the set $\mathcal{V}$.

The process $Y_{\epsilon}(t)$ is Markovian provided the initial filtration is large enough. Indeed, denote by $\left\{\mathcal{F}_{t}^{0}: t \geq 0\right\}$ the natural filtration of $C\left(\mathbb{R}_{+}, \mathbb{T}^{d}\right): \mathcal{F}_{t}^{0}=\sigma\left(X_{s}: 0 \leq s \leq t\right)$. Fix $x_{0} \in \mathcal{V}$ and denote by $\left\{\mathcal{F}_{t}: t \geq 0\right\}$ the usual augmentation of $\left\{\mathcal{F}_{t}^{0}: t \geq 0\right\}$ with respect to $\mathbb{P}_{x_{0}}^{\epsilon}$. We refer to Section III.9 of [34] for a precise definition, and to [29] for a proof of the next result which relies on the identity (5.4).

Lemma 5.3. For each $t \geq 0, S_{\mathcal{V}}(t)$ is a stopping time with respect to the filtration $\left\{\mathcal{F}_{t}\right\}$.

As $S_{\mathcal{V}}(t)$ is a stopping time with respect to the filtration $\left\{\mathcal{F}_{t}\right\}, Y_{\epsilon}(t)$ is a $\mathcal{V}$-valued, Markov process with respect to the filtration $\mathcal{G}_{t}:=\mathcal{F}_{S(t)}$. Let $\Psi: \mathcal{V} \rightarrow S$ be the projection given by

$$
\Psi(x)=\sum_{j=1}^{n} j \chi v_{j}(x),
$$

and denote by $y_{\epsilon}(t)$ the $S$-valued process obtained by projecting $Y_{\epsilon}(t)$ with $\Psi$ :

$$
y_{\epsilon}(t)=\Psi\left(Y_{\epsilon}(t)\right) .
$$

Note that the process $y_{\epsilon}(t)$ is not Markovian.

Denote by $\mathbb{Q}_{x}^{\epsilon}$, resp. $\mathbf{Q}_{x}^{\epsilon}, x \in \mathcal{V}$, the probability measure on $D\left(\mathbb{R}_{+}, \mathcal{V}\right)$, resp. $D\left(\mathbb{R}_{+}, S\right)$, induced by the process $Y_{\epsilon}(t)$, resp. $y_{\epsilon}(t)$, given that $Y_{\epsilon}(0)=x$. Fix $j \in S, x \in \mathcal{V}_{j}$. As usual, the proof that $\mathbf{Q}_{x}^{\epsilon}$ converges to $\mathbf{Q}_{j}$ is divided in two steps. We first show that the sequence $\mathbf{Q}_{x}^{\epsilon}$ is tight and then we prove the uniqueness of limit points.

Lemma 5.4. Assume that conditions (5.3) is in force. Suppose, furthermore, that

$$
\lim _{r \rightarrow 0} \limsup _{\epsilon \rightarrow 0} \max _{1 \leq j \leq n} \sup _{x \in \mathcal{V}_{j}} \mathbb{P}_{x}^{\epsilon}\left[H\left(\breve{\mathcal{V}}_{j}\right) \leq r \theta_{\epsilon}\right]=0 .
$$

Then, for every $1 \leq j \leq n, x_{0} \in \mathcal{V}_{j}$, the sequence of measures $\mathbf{Q}_{x_{0}}^{\epsilon}$ is tight. Moreover, every limit point $\mathbf{Q}^{*}$ of the sequence $\mathbf{Q}_{x_{0}}^{\epsilon}$ is such that

$$
\mathbf{Q}^{*}\{x: x(0)=j\}=1 \text { and } \mathbf{Q}^{*}\{x: x(t) \neq x(t-)\}=0
$$

for every $t>0$.

A proof of this result for one-dimensional diffusions is presented in [29, Lemma 7.5]. Condition (5.5) asserts that in the time-scale $\theta_{\epsilon}$, the process $X_{t}^{\epsilon}$ may not jump instantaneously from one well to the other. We show in Section 8 of this article that the probability $\mathbb{P}_{x}^{\epsilon}\left[H\left(\breve{\mathcal{V}}_{j}\right) \leq r \theta_{\epsilon}\right]$ is bounded by the capacity between two sets for an enlarged process. The proof of this lemma is thus reduced to an estimate of capacities. 
The proof of uniqueness relies on the characterization of continuous-time Markov chains as solutions of martingale problems. One needs to show that

$$
\boldsymbol{F}(y(t))-\int_{0}^{t}(\boldsymbol{L F})(y(s)) d s
$$

is a martingale under $\mathbf{Q}$ for all functions $\boldsymbol{F}: S \rightarrow \mathbb{R}$ and all limit point $\mathbf{Q}$ of the sequence $\mathbf{Q}_{x_{0}}^{\epsilon}$.

We proved in [3, 5] that this property is in force in the context of countable state spaces provided the mean jump rates converge and if each well $\mathcal{V}_{j}$ has an element $z_{j}$ such that

$$
\lim _{\epsilon \rightarrow 0} \sup _{y \in \mathcal{V}_{j}, y \neq z_{j}} \frac{\operatorname{cap}_{\epsilon}\left(\mathcal{V}_{j}, \breve{\mathcal{V}}_{j}\right)}{\operatorname{cap}_{\epsilon}\left(\{y\},\left\{z_{j}\right\}\right)}=0 .
$$

The point $z_{j}$ is not special. Typically, if (5.8) holds for a point $z_{j}$ in the well, it holds for all the other ones. We refer to [3, 5] for details.

Condition (5.8) has been derived for Markov processes which "visit points", that is, for Markov processes which visit all points of a well before reaching another well. This is the case of condensing zero-range processes [4, 24], random walks in potential fields [26, 28], one-dimensional diffusions [29], and for all processes whose wells are reduced to singletons, as the inclusion processes [7].

We present here an alternative method to deduce (5.7) which relies on certain asymptotic properties of the elliptic operator $\mathcal{L}_{\epsilon}$. Fix a function $\boldsymbol{F}: S \rightarrow \mathbb{R}$, let $\boldsymbol{G}=\boldsymbol{L F}$, and let $g: \mathbb{T}^{d} \rightarrow \mathbb{R}$ be given by

$$
g=\sum_{i=1}^{n} \boldsymbol{G}(i) \chi_{v_{i}}
$$

Assume that there exists a sequence of function $g_{\epsilon}: \mathbb{T}^{d} \rightarrow \mathbb{R}$ such that

(P1) $g_{\epsilon}$ vanishes on $\mathcal{V}^{c}$ and converges to $g$ uniformly on $\mathcal{V}$;

(P2) The Poisson equation $\widehat{\mathcal{L}}_{\epsilon} f=g_{\epsilon}$ in $\mathbb{T}^{d}$ has a solution denoted by $f_{\epsilon}$. Moreover, there exists a finite constant $C_{0}$ such that

$$
\sup _{0<\epsilon<1} \sup _{x \in \mathbb{T}^{d}}\left|f_{\epsilon}(x)\right| \leq C_{0}, \quad \text { and } \lim _{\epsilon \rightarrow 0} \sup _{x \in \mathcal{V}}\left|f_{\epsilon}(x)-f(x)\right|=0,
$$

where $f: \mathbb{T}^{d} \rightarrow \mathbb{R}$ is given by $f=\sum_{1 \leq i \leq n} \boldsymbol{F}(i) \chi_{\mathcal{V}_{i}}$.

The natural candidate for $g_{\epsilon}$ in conditions (P1) and (P2) is the function $g$ itself. However, as the process is ergodic, the Poisson equation $\widehat{\mathcal{L}}_{\epsilon} f=b$ has a solution only if $b$ has mean zero with respect to $\mu_{\epsilon}$. We need therefore to modify $g$ to obtain a mean-zero function. Denote by $\pi$ the stationary state of the Markov chain whose generator is $\boldsymbol{L}$. We expect $\mu_{\epsilon}\left(\mathcal{V}_{i}\right)$ to converge to $\pi_{i}$. Hence,

$$
\lim _{\epsilon \rightarrow 0} E_{\mu_{\epsilon}}[g]=\lim _{\epsilon \rightarrow 0} \sum_{i=1}^{n} \boldsymbol{G}(i) \mu_{\epsilon}\left(\mathcal{V}_{i}\right)=\sum_{i=1}^{n} \boldsymbol{L} \boldsymbol{F}(i) \pi_{i}=0 .
$$

A reasonable candidate for $g_{\epsilon}$ is thus $g-r(\epsilon) \chi \mathcal{V}_{1}$, where $r(\epsilon)=E_{\mu_{\epsilon}}[g] / \mu_{\epsilon}\left(\mathcal{V}_{1}\right)$.

Properties (P1), (P2) have been proved in [13, 35] for elliptic operators on $\mathbb{R}^{d}$ of the form $\mathcal{L}_{\epsilon} f=e^{V / \epsilon} \nabla \cdot\left(e^{-V / \epsilon} a \nabla f\right)$ and in [29] for one-dimensional diffusions with periodic boundary conditions. It is an open problem to prove these conditions in the context of interacting particle systems.

Lemma 5.5. Fix $1 \leq j \leq n$ and $x_{0} \in \mathcal{V}_{j}$. Assume that conditions (P1) and (P2) are in force. Let $\mathbf{Q}^{*}$ be a limit point of the sequence $\mathbf{Q}_{x_{0}}^{\epsilon}$ satisfying (5.6). Then, for every $\boldsymbol{F}: S \rightarrow \mathbb{R}$, 5.7) is a martingale under the measure $\mathbf{Q}^{*}$. 
Proof. Fix $1 \leq j \leq n, x_{0} \in \mathcal{V}_{j}$ and a function $\boldsymbol{F}: S \rightarrow \mathbb{R}$. Let $f_{\epsilon}: \mathbb{T}^{d} \rightarrow \mathbb{R}$ be the function given by assumption (P2). Then,

$$
M_{\epsilon}(t)=f_{\epsilon}\left(\widehat{X}_{t}^{\epsilon}\right)-\int_{0}^{t}\left(\widehat{\mathcal{L}}_{\epsilon} f_{\epsilon}\right)\left(\widehat{X}_{s}^{\epsilon}\right) d s=f_{\epsilon}\left(\widehat{X}_{t}^{\epsilon}\right)-\int_{0}^{t} g_{\epsilon}\left(\widehat{X}_{s}^{\epsilon}\right) d s
$$

is a martingale with respect to the filtration $\mathcal{F}_{t}$ and the measure $\widehat{\mathbb{P}}_{x_{0}}^{\epsilon}$. Since $\left\{S_{\mathcal{V}}(t): t \geq 0\right\}$ are stopping times with respect to $\mathcal{F}_{t}$,

$$
\widehat{M}_{\epsilon}(t)=M_{\epsilon}\left(S_{\mathcal{V}}(t)\right)=f_{\epsilon}\left(Y_{\epsilon}(t)\right)-\int_{0}^{S_{\mathcal{V}}(t)} g_{\epsilon}\left(\widehat{X}_{s}^{\epsilon}\right) d s
$$

is a martingale with respect to the filtration $\mathcal{G}_{t}$. Since $g_{\epsilon}$ vanishes on $\mathcal{V}^{c}$, by a change of variables,

$$
\int_{0}^{S_{\mathcal{V}}(t)} g_{\epsilon}\left(\widehat{X}_{s}^{\epsilon}\right) d s=\int_{0}^{S_{\mathcal{V}}(t)} g_{\epsilon}\left(\widehat{X}_{s}^{\epsilon}\right) \chi_{\mathcal{V}}\left(\widehat{X}_{s}^{\epsilon}\right) d s=\int_{0}^{t} g_{\epsilon}\left(\widehat{X}^{\epsilon}\left(S_{\mathcal{V}}(s)\right)\right) d s .
$$

Hence,

$$
\widehat{M}_{\epsilon}(t)=f_{\epsilon}\left(Y_{\epsilon}(t)\right)-\int_{0}^{t} g_{\epsilon}\left(Y_{\epsilon}(s)\right) d s
$$

is a $\left\{\mathcal{G}_{t}\right\}$-martingale under the measure $\mathbb{Q}_{x_{0}}^{\epsilon}$.

By (P1) and (P2), $g_{\epsilon}$, resp. $f_{\epsilon}$, converge to $g$, resp. $f$, uniformly in $\mathcal{V}$ as $\epsilon \rightarrow 0$. Hence, since $Y_{\epsilon}(s) \in \mathcal{V}$ for all $s \geq 0$, we may replace in the previous equation $g_{\epsilon}, f_{\epsilon}$ by $g, f$, respectively, at a cost which vanishes as $\epsilon \rightarrow 0$. Therefore,

$$
\widehat{M}_{\epsilon}(t)=f\left(Y_{\epsilon}(t)\right)-\int_{0}^{t} g\left(Y_{\epsilon}(s)\right) d s+o(1)
$$

is a $\left\{\mathcal{G}_{t}\right\}$-martingale under the measure $\mathbb{Q}_{x_{0}}^{\epsilon}$.

Since $f$ and $g$ are constant on each set $\mathcal{V}_{i}, f\left(Y_{\epsilon}(t)\right)=\boldsymbol{F}\left(y_{\epsilon}(t)\right), g\left(Y_{\epsilon}(t)\right)=\boldsymbol{G}\left(y_{\epsilon}(t)\right)$. By the second condition in (5.6), $\mathbf{Q}^{*}$ is concentrated on trajectories which are continuous at any fixed time with probability 1 . We may, therefore, pass to the limit and conclude that $\boldsymbol{F}(y(t))-\int_{0}^{t}(\boldsymbol{L} \boldsymbol{F})(y(s)) d s$ is a martingale under $\mathbf{Q}^{*}$.

Theorem 5.6. Assume that conditions $(P 1),(P 2),(5.3)$, (5.5) are in force. Fix $j \in S$ and $x_{0} \in \mathcal{V}_{j}$. The sequence of measures $\mathbf{Q}_{x_{0}}^{\epsilon}$ converges, as $\epsilon \rightarrow 0$, to the probability measure $\mathbf{Q}_{j}$.

Proof. The assertion is a consequence of Lemma 5.4, Lemma 5.5 and the fact that there is only one measure $\mathbf{Q}$ on $D\left(\mathbb{R}_{+}, S\right)$ such that $\mathbf{Q}[x(0)=j]=1$ and such that (5.7) is a martingale for all $\boldsymbol{F}: S \rightarrow \mathbb{R}$.

Step 4: The finite-dimensional distributions. By [25, Proposition 1.1], the finite-dimensional distributions of $x_{\epsilon}(t)$ converge to the finite-dimensional distributions of $y(t)$ if the process $y_{\epsilon}(t)$ converges in the Skorohod topology to $y(t)$ [Theorem 5.6], if in the timescale $\theta_{\epsilon}$ the total time spent in $\Delta$ is negligible [Lemma 5.2] and if

$$
\lim _{\delta \rightarrow 0} \limsup _{\epsilon \rightarrow 0} \sup _{x \in \mathcal{V}} \sup _{\delta \leq s \leq 2 \delta} \mathbb{P}_{x}^{\epsilon}\left[X^{\epsilon}\left(s \theta_{\epsilon}\right) \in \Delta\right]=0 .
$$

This completes the argument. The convergence of the finite-dimensional distributions of $X_{t}^{\epsilon}$ and sharp asymptotics for the transition time in the context of diffusions were first obtained by Sugiura [37, 38]. The approach presented in this section to prove the metastable 
behavior of a Markov process has been proposed by Beltrán and L. [3, 5]. It has been successfully applied to many models quoted in this section. For further reading on metastability, we refer to the books by Olivieri and Vares [30] and Bovier and den Hollander [11].

Acknowledgments. The results presented in this review are the outcome of long standing collaborations. The author wishes to thank his colleagues and friends, J. Beltrán, E. Chavez, A. Gaudillière, M. Jara, M. Loulakis, M. Mariani, R. Misturini, M. Mourragui, I. Seo, A. Teixeira, K. Tsunoda.

\section{REFERENCES}

[1] S. Arrhenius: On the reaction velocity of the inversion of cane sugar by acids. J. Phys. Chem. 4, 226 (1889). Partially translated to english in "Selected readings in chemical kinetics" edited by M. Back and K. Laidler, Pergamon press, Oxford 1967.

[2] J. Beltrán, E. Chavez, C. Landim: From coalescing random walks on a torus to Kingman's coalescent. preprint (2017).

[3] J. Beltrán, C. Landim: Tunneling and metastability of continuous time Markov chains. J. Stat. Phys. 140, 1065-1114, (2010).

[4] J. Beltrán, C. Landim: Metastability of reversible condensed zero range processes on a finite set. Probab. Th. Rel. Fields 152, 781-807 (2012).

[5] J. Beltrán, C. Landim: Tunneling and metastability of continuous time Markov chains II. J. Stat. Phys. 149, 598-618, (2012).

[6] N. Berglund: Kramers' law : validity, derivations and generalisations. Markov Process. Related Fields, 19, 459-490 (2013).

[7] A. Bianchi, S. Dommers, C. Giardinà: Metastability in the reversible inclusion process. Elect. J. Probab. 22, 70 (2017)

[8] F. Bouchet, J. Reygner: Generalisation of the Eyring-Kramers transition rate formula to irreversible diffusion processes. preprint (2015) http://arxiv.org/abs/1507.02104

[9] A. Bovier, M. Eckhoff, V. Gayrard, M. Klein: Metastability in stochastic dynamics of disordered mean-field models. Probab. Theory Relat. Fields 119, 99-161 (2001)

[10] A. Bovier, M. Eckhoff, V. Gayrard, M. Klein: Metastability in reversible diffusion process I. Sharp asymptotics for capacities and exit times. J. Eur. Math. Soc. 6, 399-424 (2004).

[11] A. Bovier, F. den Hollander: Metastability: a potential-theoretic approach. Grundlehren der mathematischen Wissenschaften 351, Springer, Berlin, 2015.

[12] P. Doyle: Energy for Markov chains. Unpublished manuscript available at http://www.math.dartmouth.edu/d̃oyle (1994).

[13] L. C. Evans, P. R. Tabrizian: Asymptotic for scaled Kramers-Smoluchowski equations. SIAM J. Math. Anal. 48, 2944-2961 (2016)

[14] H. Eyring: The activated complex in chemical reactions. J. Chem. Phys. 3, 107-115 (1935).

[15] M. I. Freidlin, A. D. Wentzell: Random perturbations of dynamical systems. Second edition. Grundlehren der Mathematischen Wissenschaften [Fundamental Principles of Mathematical Sciences], 260. SpringerVerlag, New York, 1998.

[16] A. Gaudillière, C. Landim: A Dirichlet principle for non reversible Markov chains and some recurrence theorems. Probab. Theory Related Fields 158, 55-89 (2014).

[17] D. Gilbarg, N. S. Trudinger. Elliptic partial differential equations of second order. Springer, 2015.

[18] J. van't Hoff: Studies in Chemical Dynamics Chemical publishing Company, 1896.

[19] J. Hood. Phil. Mag. 6, 371 (1878) and 20, 323 (1885).

[20] M. Jara, C. Landim, A. Teixeira: Quenched scaling limits of trap models. Ann. Probab. 39, 176-223 (2011).

[21] M. Jara, C. Landim, A. Teixeira: Universality of trap models in the ergodic time scale. Ann. Probab. 42, 2497-2557 (2014).

[22] O. D. Kellogg. Foundations of potential theory. Dover Publications Inc., New York, 1954.

[23] H. A. Kramers: Brownian motion in a field of force and the diffusion model of chemical reactions. Physica 7, 284-304, (1940).

[24] C. Landim; Metastability for a non-reversible dynamics: the evolution of the condensate in totally asymmetric zero range processes. Commun. Math. Phys. 330, 1-32 (2014).

[25] C. Landim, M. Loulakis, M. Mourragui: Metastable Markov chains. arXiv:1703.09481 (2017).

[26] C. Landim, R. Misturini, K. Tsunoda; Metastability of reversible random walks in potential fields. J. Stat. Phys. 160 1449-1482 (2015). 
[27] C. Landim, M. Mariani, I. Seo: A Dirichlet and a Thomson principle for non-selfadjoint elliptic operators, metastability in non-reversible diffusion processes. arXiv:1701.00985 (2017).

[28] C. Landim, I. Seo: Metastability of non-reversible random walks in a potential field, the Eyring-Kramers transition rate formula. To appear in Commun. Pure Appl. Math. arXiv:1605.01009 (2016).

[29] C. Landim, I. Seo: Metastability of one-dimensional, non-reversible diffusions with periodic boundary conditions. arXiv:1710.06672 (2017).

[30] E. Olivieri, M. E. Vares: Large deviations and metastability. In: Encyclopedia of Mathematics and its Applications, vol. 100. Cambridge University Press, Cambridge 2005.

[31] R. G. Pinsky: A generalized Dirichlet principle for second order nonselfadjoint elliptic operators. SIAM J. Math. Anal. 19, 204-213 (1988).

[32] R. G. Pinsky: A minimax variational formula giving necessary and sufficient conditions for recurrence or transience of multidimensional diffusion processes. Ann. Probab. 16, 662-671 (1988).

[33] R. G. Pinsky. Positive harmonic functions and diffusion Cambridge studies in advanced mathematics Vol. 45. Cambridge University Press, 1995.

[34] L. C. G. Rogers, D. Williams: Diffusions, Markov Processes, and Martingales: Volume 1, Foundations. Cambridge University Press, 1994.

[35] I. Seo, P. R. Tabrizian: Asymptotics for scaled Kramers-Smoluchowski equations in several dimensions with general potentials. preprint (2017).

[36] M. Slowik: A note on variational representations of capacities for reversible and nonreversible Markov chains. unpublished, Technische Universität Berlin, 2012.

[37] M. Sugiura: Metastable behaviors of diffusion processes with small parameter. J. Math. Soc. Japan 47, 755-788 (1995).

[38] M. Sugiura: Asymptotic behaviors on the small parameter exit problems and the singularly perturbation problems. Ryukyu Math. J. 14, 79-118 (2001).

IMPA, ESTRADA DONA CASTORINA 110, CEP 22460 Rio DE JANEIRO, BRASIL AND CNRS UMR 6085, Université de Rouen, AVEnUe de L'Université, BP.12, TeChNopôle du MAdRILlet, F7680 1 SAINT-ÉTIENNE-DU-ROUVRAY, FRANCE.

E-MAIL: landim@impa.br 